\title{
Neonatal Outcomes of Infants Admitted to a Large Government Hospital in Amman, Jordan
}

\author{
Priya G. Sivasubramaniam ${ }^{1}$, Cristin E. Quinn ${ }^{1}$, Meridith Blevins ${ }^{2,3}$, Ahmand Al Hajajra ${ }^{5}$, Najwa Khuri-Bulos ${ }^{6}$, \\ Samir Faouri ${ }^{5} \&$ Natasha Halasa ${ }^{2,4}$ \\ ${ }^{1}$ Vanderbilt University School of Medicine, Nashville, TN, USA \\ ${ }^{2}$ Vanderbilt Institute for Global Health, 2525 West End Avenue Suite 750, Nashville, TN, 37203, USA \\ ${ }^{3}$ Departments of Biostatistics, Vanderbilt University School of Medicine, Nashville, TN, USA \\ ${ }^{4}$ Pediatrics, Vanderbilt University School of Medicine, Nashville, TN, USA \\ ${ }^{5}$ Pediatrics, Al-Bashir Government Hospital, Amman, Jordan \\ ${ }^{6}$ Pediatrics, Division of Infectious Diseases, Jordan University Hospital, Jordan \\ Correspondence: Priya G. Sivasubramaniam, Vanderbilt University School of Medicine, Nashville, TN, USA. \\ E-mail: priya.g.sivasubramaniam@Vanderbilt.Edu; Natasha Halasa, 1161 21st Ave South MCN D7232 Nashville, \\ TN 37232, USA. Tel: 1-613-947-3592. E-mail: natasha.halasa@vanderbilt.edu
}

Received: October 14, 2014 Accepted: December 17, 2014 Online Published: January 14, 2015

doi:10.5539/gjhs.v7n4p217 URL: http://dx.doi.org/10.5539/gjhs.v7n4p217

\begin{abstract}
Objective: To describe characteristics and outcomes of Jordanian newborns admitted to a large governmental neonatal intensive care unit (NICU).
\end{abstract}

Methods: Newborns born at the government hospital, Al Bashir, in Amman, Jordan were prospectively enrolled. The study focused on newborns admitted to the NICU and a retrospective chart review was performed. Abstraction included in-hospital mortality, antibiotic days, ventilation, oxygen use, and CRP levels. Rank sum and chi-squared tests were used to compare across outcomes. Logistic regression of hypothesized risk factors with death adjusted for gestational age.

Results: Of the 5,466 neonates enrolled from 2/10-2/11, medical records were available for 321/378(84.9\%) infants admitted to the NICU. The median gestational age was 36 weeks, median birth weight was $2.3 \mathrm{~kg}$, and $28(8.7 \%)$ infants died. The two most common reasons for admission and mortality were respiratory distress syndrome and prematurity. Low Apgar scores and positive CRP were predictors of mortality. Risk factors associated with increased use of antibiotics, oxygen hood, and mechanical ventilation included lower gestational age and prematurity.

Conclusion: Infants admitted to the Jordanian NICU have significantly higher median gestational age and birth weights than in developed countries and were associated with significant morbidity and mortality. Continuations of global efforts to prevent prematurity are needed.

Keywords: Jordan, Middle East, mortality, neonatal intensive care unit (NICU), outcomes, prematurity

\section{Introduction}

\subsection{Introduction}

Much is known regarding outcomes of neonates admitted to the neonatal intensive care unit (NICU) in Western countries. Internationally, major causes of neonatal mortality in the NICU include birth before 37 weeks gestation (28\%), infections (26\%), and asphyxia (23\%). In Western countries such as the United States, congenital malformations account for a significant cause of neonatal mortality (20\%). Over the past three decades, global mortality rates of NICU neonates have dramatically improved due to the introduction of surfactant, steroids, and perinatal care, thereby enabling improved survival of very low birth weight infants as young as 23 to 28 weeks. As a result, neonates in countries with data regarding neonatal outcomes tend to be admitted to the NICU as young as 22 weeks for treatment, with survival rates continuing to ascend (Feng, 2011; Simpson, Xiang, Hellmann, \& Tomlinson, 2010). Nonetheless, neonatal mortality continues to constitute $44 \%$ of childhood deaths under the age of five (WHO, 2013). 


\subsection{Significance}

In developing countries such as those in the Middle East, data regarding NICU outcomes are sparse. Admission data, trends of mortality rates, causes of death, and NICU treatments, for instance, are unknown. While developed nations have reduced the neonatal mortality rate by 54\% from 1990 to 2012, developing nations have only seen a $37 \%$ reduction in this rate, with some regions as low as $17 \%$ (WHO, 2013). Given the currently unmet United Nations Millennium Development Goal to reduce child mortality by two thirds between 1990 and 2015 and the majority of these deaths occurring in developing countries including and surrounding those of the Middle East, it is necessary report neonatal outcome data in this region (UN, 2010). To date, no comprehensive study has focused on a large, representative hospital in a Middle Eastern nation reporting general admission and outcome NICU characteristics.

\subsection{Hypotheses and Their Correspondence to Research Design}

Our group hypothesized that we would find significant differences in clinical outcomes between neonates in Western nations compared to those of a representative developing nation within the Middle East. Therefore, our group took advantage of an already existing neonatal cohort of newborns enrolled prospectively at Al-Bashir Hospital, a large government-funded hospital in Amman, Jordan, which serves as a primary hospital for the nation (Khuri-Bulos et al., 2013). The aim of our study was to determine neonatal outcome of newborns admitted to the NICU and risk factors for mortality.

\section{Methods and/or Techniques}

\subsection{Study Design and Participants}

As part of a larger study, infants born at Al Bashir hospital were prospectively enrolled at birth for a one-time assessment of their vitamin D levels (Khuri-Bulos et al., 2013). Verbal consent was obtained from mothers to obtain heel sticks for blood from their infants. A brief questionnaire assessing demographic and social behaviors of the mothers was used. All infants enrolled within 96 hours of birth were eligible for enrollment. From this cohort, neonates admitted to the NICU were identified and chart review was then performed. Al Bashir is one of three major hospitals serving Amman, Jordan. The University of Jordan, the Institutional Review Boards of Vanderbilt University, and the Jordanian Ministry of Health at Al Bashir Hospital approved this study.

\subsection{Questionnaires}

The research team used a standardized case report questionnaire. Parents were asked to provide nationality of mother and father, child's date of birth, route of delivery, child's birth weight, mother's vitamin D supplementation history, daily number of hours that mother spends outdoors, mother's clothing practice, whether or not the mother smoked during her pregnancy (and if so, which of the trimesters), and if the mother was exposed to smoke in her household during pregnancy. A database was implemented using REDCap (Research Electronic Data Capture), a secure, web-based application designed to support data capture for research studies, created and hosted at Vanderbilt University (Harris, Taylor, Thielke, Payne, Gonzalez, \& Conde, 2009).

\subsection{Clinical Outcomes Measures}

Data were abstracted from the clinical charts of the neonates admitted to the NICU to collect admission and outcome measures. The major outcomes of interested included: in-hospital mortality, antibiotic days, mechanical ventilation, oxygen use, and elevated (c-reactive protein) CRP levels. In-hospital mortality referred to infant death prior to NICU discharge. Antibiotic days referred to the total number of days on all antibiotics, with information regarding specific antibiotics used. Mechanical ventilation and oxygen use as well as total number of days were calculated. Elevated CRP was defined as having a CRP above a set value at any point during the infant's hospitalization in the NICU.

\subsection{Statistical Analyses}

Descriptive statistics were used to summarize sociodemographic and clinical characteristics for each aforementioned NICU outcome, and tests of association included Wilcoxon rank sum and chi-square tests. The dependence of duration of antibiotic use on gestational age, sepsis, RDS, birth weight, Apgar score, vitamin D, premature rupture of membranes (PROM), preeclampsia, and positive CRP was modeled using negative binomial regression; only babies who were alive at discharge were included due to truncation by death. Due to a limited number of deaths, 11 different logistic regression models were estimated for a set of 11 covariates while adjusting for gestational age to prevent over-fitting a larger model. To account for possible non-linear associations, continuous variables were included using restricted cubic splines. Model covariates were identified a priori and no adjustment was made for multiple comparisons. We employed R-software 2.15.1 
(www.r-project.org) for all data analyses. Analysis scripts are available at http://biostat.mc.vanderbilt.edu/ArchivedAnalyses.

\section{Results}

\subsection{Patient Population Characteristics and Recruitment}

From February 2010 to July 2011, 378 out of 5,466 neonates in our prospective cohort were admitted to the NICU. Of these 378 infants, medical records were available for 321 (85\%). Median gestational age of neonates admitted to the NICU was 36 weeks (interquartile range [IQR] 34-37 weeks), median birth weight was $2.3 \mathrm{~kg}$ (IQR 1.9-2.9 kg), and $154(48 \%)$ of these neonates were female. Median length of stay in the NICU was 4 days (range 2-8 days) and the two most common reasons for admission were respiratory distress syndrome $(67 \%)$ and prematurity $(52 \%)$. Table 1 provides a summary of patient population characteristics.

Table 1. NICU Characteristics by Vital Status at Discharge, Oxyhood, and Ventilator Use

Vital Status

\begin{tabular}{|c|c|c|c|c|}
\hline & $\begin{array}{l}\text { Dead } \\
(n=28)\end{array}$ & $\begin{array}{l}\text { Alive } \\
(n=293)\end{array}$ & $\begin{array}{l}\text { Combined } \\
(n=321)\end{array}$ & P-value \\
\hline Female & $14(50 \%)$ & $140(48 \%)$ & $154(48 \%)$ & 0.98 \\
\hline Gestational age & $30(29-33)$ & $36(35-37)$ & $36(34-37)$ & $<0.001$ \\
\hline Missing, $\mathrm{n}(\%)$ & $0(0 \%)$ & $4(1 \%)$ & $4(1 \%)$ & \\
\hline Days in hospital & $6(2-11)$ & $4(3-8)$ & $4(2-8)$ & 0.65 \\
\hline \multicolumn{5}{|l|}{ Reason(s) for admission } \\
\hline Sepsis & $6(21 \%)$ & $25(9 \%)$ & $31(10 \%)$ & 0.061 \\
\hline RDS & $24(86 \%)$ & $191(65 \%)$ & $215(67 \%)$ & 0.046 \\
\hline Neonatal pneumonia & $1(4 \%)$ & $0(0 \%)$ & $1(<1 \%)$ & 0.14 \\
\hline Prematurity & $21(75 \%)$ & $146(50 \%)$ & $167(52 \%)$ & 0.019 \\
\hline Heart Disease & $2(7 \%)$ & $4(1 \%)$ & $6(2 \%)$ & 0.15 \\
\hline Congenital Malformation & $4(14 \%)$ & $14(5 \%)$ & $18(6 \%)$ & 0.097 \\
\hline Overweight Infant & $0(0 \%)$ & $17(6 \%)$ & $17(5 \%)$ & 0.39 \\
\hline Low birth weight infant & $3(11 \%)$ & $42(14 \%)$ & $45(14 \%)$ & 0.81 \\
\hline Jaundice & $0(0 \%)$ & $16(5 \%)$ & $16(5 \%)$ & 0.42 \\
\hline Low Apgar score & $4(14 \%)$ & $15(5 \%)$ & $19(6 \%)$ & 0.12 \\
\hline Seizures & $0(0 \%)$ & $1(<1 \%)$ & $1(<1 \%)$ & 0.99 \\
\hline Hypoglycemia & $0(0 \%)$ & $4(1 \%)$ & $4(1 \%)$ & 0.99 \\
\hline Neonatal Asphyxia & $3(11 \%)$ & $11(4 \%)$ & $14(4 \%)$ & 0.22 \\
\hline Other & $5(18 \%)$ & $94(32 \%)$ & $99(31 \%)$ & 0.18 \\
\hline Birth weight (kg) & $1.2(0.9-1.9)$ & $2.3(2-2.9)$ & $2.3(1.9-2.9)$ & $<0.001$ \\
\hline Missing, $\mathrm{n}(\%)$ & $0(0 \%)$ & $1(<1 \%)$ & $1(<1 \%)$ & \\
\hline Weight at 5 days (kg) & $1.3(1.2-1.6)$ & $\begin{array}{l}2.07(1.785- \\
2.65)\end{array}$ & $2(1.73-2.6)$ & $<0.001$ \\
\hline Missing, $\mathrm{n}(\%)$ & $19(68 \%)$ & $194(66 \%)$ & $213(66 \%)$ & \\
\hline Apgar at 1 minute & $4(3-5)$ & $7(6-7)$ & $7(5-7)$ & $<0.001$ \\
\hline Missing, $\mathrm{n}(\%)$ & $7(25 \%)$ & $135(46 \%)$ & $142(44 \%)$ & \\
\hline Apgar at 5 minutes & $6(5-7.5)$ & $8(7-8)$ & $8(7-8)$ & $<0.001$ \\
\hline Missing, $\mathrm{n}(\%)$ & $5(18 \%)$ & $113(39 \%)$ & $118(37 \%)$ & \\
\hline CRP (ever positive), $\mathrm{n}(\%)$ & & & & $<0.001$ \\
\hline Missing & $9(32 \%)$ & $21(7 \%)$ & $30(9 \%)$ & \\
\hline Negative & $12(63 \%)$ & $255(94 \%)$ & $267(92 \%)$ & \\
\hline
\end{tabular}




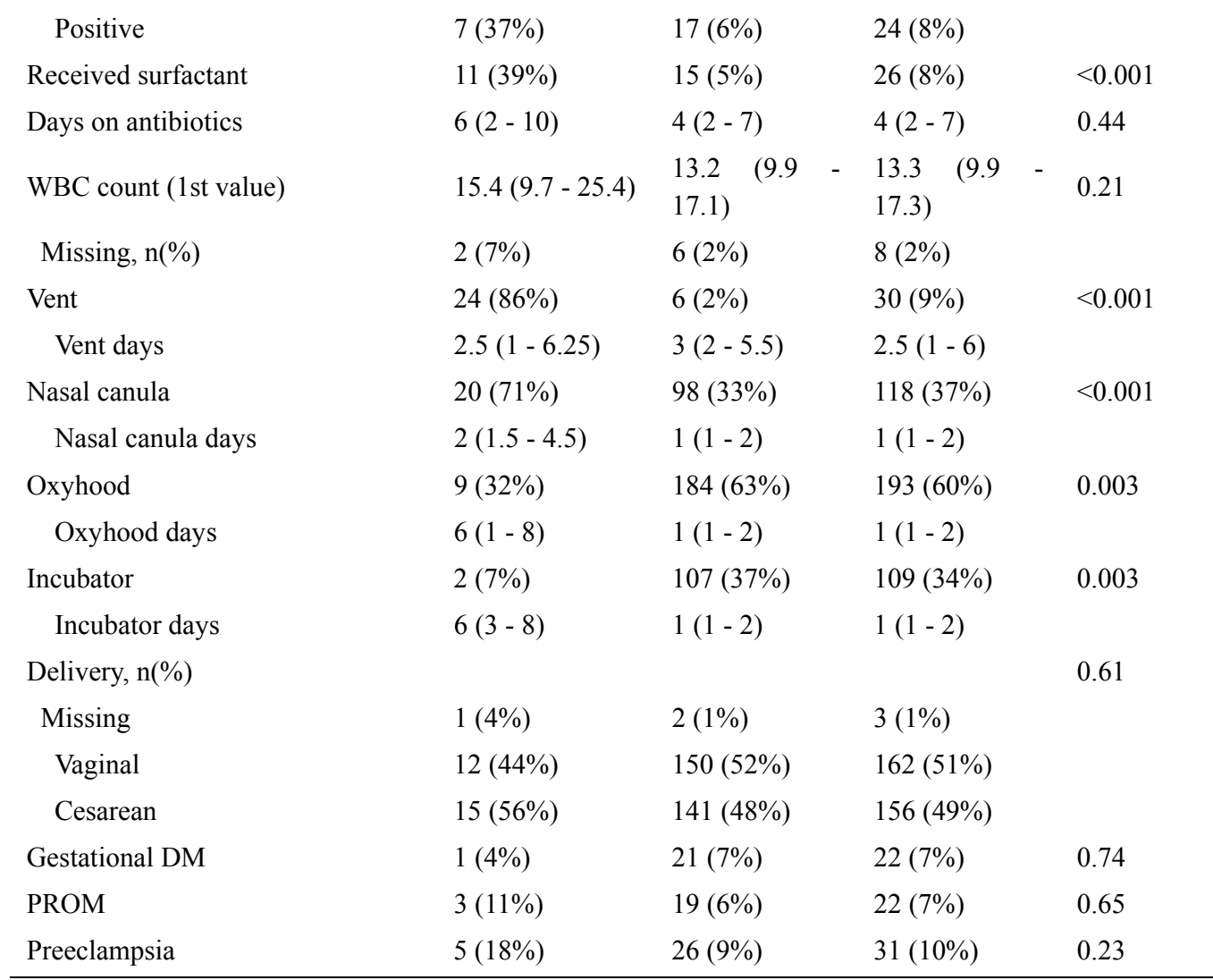

Oxyhood use

\begin{tabular}{|c|c|c|c|c|}
\hline & $\begin{array}{l}\text { Oxyhood } \\
(n=193)\end{array}$ & $\begin{array}{l}\text { No Oxyhood } \\
(n=128)\end{array}$ & $\begin{array}{l}\text { Combined } \\
(n=321)\end{array}$ & P-value \\
\hline Female & $91(47 \%)$ & $63(49 \%)$ & $154(48 \%)$ & 0.80 \\
\hline Gestational age & $35(34-37)$ & $37(35-37)$ & $36(34-37)$ & 0.002 \\
\hline Missing, $\mathrm{n}(\%)$ & $0(0 \%)$ & $4(3 \%)$ & $4(1 \%)$ & \\
\hline Days in hospital & $5(3-9)$ & $3(2-6)$ & $4(2-8)$ & $<0.001$ \\
\hline \multicolumn{5}{|l|}{ Reason(s) for admission } \\
\hline Sepsis & $14(7 \%)$ & $17(13 \%)$ & $31(10 \%)$ & 0.11 \\
\hline RDS & $159(82 \%)$ & $56(44 \%)$ & $215(67 \%)$ & $<0.001$ \\
\hline Neonatal pneumonia & $0(0 \%)$ & $1(1 \%)$ & $1(<1 \%)$ & 0.84 \\
\hline Prematurity & $118(61 \%)$ & $49(38 \%)$ & $167(52 \%)$ & $<0.001$ \\
\hline Heart Disease & $2(1 \%)$ & $4(3 \%)$ & $6(2 \%)$ & 0.35 \\
\hline Congenital Malformation & $11(6 \%)$ & $7(5 \%)$ & $18(6 \%)$ & 0.99 \\
\hline Overweight Infant & $5(3 \%)$ & $12(9 \%)$ & $17(5 \%)$ & 0.016 \\
\hline Low birth weight infant & $27(14 \%)$ & $18(14 \%)$ & $45(14 \%)$ & 0.99 \\
\hline Jaundice & $8(4 \%)$ & $8(6 \%)$ & $16(5 \%)$ & 0.56 \\
\hline Low Apgar score & $12(6 \%)$ & $7(5 \%)$ & $19(6 \%)$ & 0.97 \\
\hline Seizures & $0(0 \%)$ & $1(1 \%)$ & $1(<1 \%)$ & 0.84 \\
\hline Hypoglycemia & $1(1 \%)$ & $3(2 \%)$ & $4(1 \%)$ & 0.35 \\
\hline Neonatal Asphyxia & $8(4 \%)$ & $6(5 \%)$ & $14(4 \%)$ & 0.99 \\
\hline Other & $44(23 \%)$ & $55(43 \%)$ & $99(31 \%)$ & $<0.001$ \\
\hline Death & $9(5 \%)$ & $19(15 \%)$ & $28(9 \%)$ & \\
\hline
\end{tabular}




\begin{tabular}{|c|c|c|c|c|}
\hline Birth weight (kg) & $2.2(1.9-2.8)$ & $2.5(2.1-3.1)$ & $2.29(1.9-2.9)$ & 0.005 \\
\hline Missing, $\mathrm{n}(\%)$ & $1(1 \%)$ & $0(0 \%)$ & $1(<1 \%)$ & \\
\hline Weight at 5 days (kg) & $1.9(1.8-2.5)$ & $2.36(1.665-2.95)$ & $2(1.7-2.6)$ & 0.32 \\
\hline Missing, $\mathrm{n}(\%)$ & $112(58 \%)$ & $101(79 \%)$ & $213(66 \%)$ & \\
\hline Apgar at 1 minute & $6(5-7)$ & $7(5-7)$ & $7(5-7)$ & 0.80 \\
\hline Missing, $\mathrm{n}(\%)$ & $79(41 \%)$ & $63(49 \%)$ & $142(44 \%)$ & \\
\hline Apgar at 5 minutes & $8(7-8)$ & $8(6-8)$ & $8(7-8)$ & 0.39 \\
\hline Missing, $\mathrm{n}(\%)$ & $66(34 \%)$ & $52(41 \%)$ & $118(37 \%)$ & \\
\hline Vitamin D & $3.5(2.5-4.8)$ & $3.5(2.5-5.3)$ & $3.5(2.5-5)$ & 0.63 \\
\hline Missing, $\mathrm{n}(\%)$ & $70(36 \%)$ & $37(29 \%)$ & $107(33 \%)$ & \\
\hline CRP (ever positive), $\mathrm{n}(\%)$ & & & & 0.82 \\
\hline Missing & $11(6 \%)$ & $19(15 \%)$ & $30(9 \%)$ & \\
\hline Negative & $168(92 \%)$ & $99(91 \%)$ & $267(92 \%)$ & \\
\hline Positive & $14(8 \%)$ & $10(9 \%)$ & $24(8 \%)$ & \\
\hline Received surfactant & $17(9 \%)$ & $9(7 \%)$ & $26(8 \%)$ & 0.717 \\
\hline Days on antibiotics & $4(3-8)$ & $3(2-5)$ & $4(2-7)$ & $<0.001$ \\
\hline WBC count (1st value) & $12.3(9.7-16.5)$ & $15(10.5-18.9)$ & $13.3(9.9-17.3)$ & 0.003 \\
\hline Missing, $\mathrm{n}(\%)$ & $4(2 \%)$ & $4(3 \%)$ & $8(2 \%)$ & \\
\hline Vent & $11(6 \%)$ & $19(15 \%)$ & $30(9 \%)$ & 0.010 \\
\hline Vent days & $3(1.5-5)$ & $2(1-6.5)$ & $2.5(1-6)$ & \\
\hline Nasal canula & $67(35 \%)$ & $51(40 \%)$ & $118(37 \%)$ & 0.42 \\
\hline Nasal canula days & $1(1-2.8)$ & $1(1-2)$ & $1(1-2)$ & \\
\hline Incubator & $84(44 \%)$ & $25(20 \%)$ & 109 (34\%) & $<0.001$ \\
\hline Incubator days & $2(1-2)$ & $1(1-2)$ & $1(1-2)$ & \\
\hline Delivery, n(\%) & & & & 0.99 \\
\hline Missing & $2(1 \%)$ & $1(1 \%)$ & $3(1 \%)$ & \\
\hline Vaginal & $97(51 \%)$ & $65(51 \%)$ & $162(51 \%)$ & \\
\hline Cesarean & $94(49 \%)$ & $62(49 \%)$ & $156(49 \%)$ & \\
\hline Gestational DM & $9(5 \%)$ & $13(10 \%)$ & $22(7 \%)$ & 0.093 \\
\hline PROM & $11(6 \%)$ & $11(9 \%)$ & $22(7 \%)$ & 0.44 \\
\hline Preeclampsia & $19(10 \%)$ & $12(9 \%)$ & $31(10 \%)$ & 0.99 \\
\hline
\end{tabular}

Mechanical Ventilation

\begin{tabular}{|c|c|c|c|c|}
\hline & $\begin{array}{l}\text { Ventilator } \\
(n=30)\end{array}$ & $\begin{array}{l}\text { No Ventilator } \\
(n=291)\end{array}$ & $\begin{array}{l}\text { Combined } \\
(\mathrm{n}=321)\end{array}$ & P-value \\
\hline Female & $17(57 \%)$ & $137(47 \%)$ & $154(48 \%)$ & 0.42 \\
\hline Gestational age & $31(29.3-354.8)$ & $36(35-37)$ & $36(34-37)$ & $<0.001$ \\
\hline Missing, $\mathrm{n}(\%)$ & $0(0 \%)$ & $4(1 \%)$ & $4(1 \%)$ & \\
\hline Days in hospital & $109.5(3-14.8)$ & $4(2-8)$ & $4(2-8)$ & 0.012 \\
\hline \multicolumn{5}{|l|}{ Reason(s) for admission } \\
\hline Sepsis & $6(20 \%)$ & $25(9 \%)$ & $31(10 \%)$ & 0.091 \\
\hline RDS & $25(83 \%)$ & $190(65 \%)$ & $215(67 \%)$ & 0.072 \\
\hline Neonatal pneumonia & $1(3 \%)$ & $0(0 \%)$ & $1(<1 \%)$ & 0.16 \\
\hline Prematurity & $21(70 \%)$ & $146(50 \%)$ & $167(52 \%)$ & 0.060 \\
\hline Heart Disease & $3(10 \%)$ & $3(1 \%)$ & $6(2 \%)$ & 0.006 \\
\hline
\end{tabular}




\begin{tabular}{|c|c|c|c|c|}
\hline Congenital Malformation & $3(10 \%)$ & $15(5 \%)$ & $18(6 \%)$ & 0.50 \\
\hline Overweight Infant & $0(0 \%)$ & $17(6 \%)$ & $17(5 \%)$ & 0.35 \\
\hline Low birth weight infant & $4(13 \%)$ & $41(14 \%)$ & $45(14 \%)$ & 0.99 \\
\hline Jaundice & $0(0 \%)$ & $16(5 \%)$ & $16(5 \%)$ & 0.38 \\
\hline Low Apgar score & $5(17 \%)$ & $14(5 \%)$ & $19(6 \%)$ & 0.027 \\
\hline Seizures & $0(0 \%)$ & $1(<1 \%)$ & $1(<1 \%)$ & 0.99 \\
\hline Hypoglycemia & $0(0 \%)$ & $4(1 \%)$ & $4(1 \%)$ & 0.99 \\
\hline Neonatal Asphyxia & $4(13 \%)$ & $10(3 \%)$ & $14(4 \%)$ & 0.040 \\
\hline Other & $7(23 \%)$ & $92(32 \%)$ & $99(31 \%)$ & 0.47 \\
\hline Death & $24(80 \%)$ & $4(1 \%)$ & $28(9 \%)$ & \\
\hline Birth weight (kg) & $1.55(1-2)$ & $2.3(2-2.9)$ & $2.29(1.9-2.9)$ & $<0.001$ \\
\hline Missing, $n(\%)$ & $0(0 \%)$ & $1(<1 \%)$ & $1(<1 \%)$ & \\
\hline Weight at 5 days $(\mathrm{kg})$ & $1.6(1.2-1.9)$ & $2.07(1.77-2.63)$ & $2(1.73-2.6)$ & 0.006 \\
\hline Missing, $n(\%)$ & $17(57 \%)$ & $196(67 \%)$ & $213(66 \%)$ & \\
\hline Apgar at 1 minute & $4(2.75-5)$ & $7(6-7)$ & $7(5-7)$ & $<0.001$ \\
\hline Missing, $\mathrm{n}(\%)$ & $6(20 \%)$ & $136(47 \%)$ & $142(44 \%)$ & \\
\hline Apgar at 5 minutes & $6(5-7)$ & $8(7-8)$ & $8(7-8)$ & $<0.001$ \\
\hline Missing, $n(\%)$ & $5(17 \%)$ & $113(39 \%)$ & $118(37 \%)$ & \\
\hline Vitamin D & $3.7(2.985-5.765)$ & $3.5(2.5-5)$ & $3.5(2.5-5)$ & 0.48 \\
\hline Missing, $n(\%)$ & $11(37 \%)$ & $96(33 \%)$ & $107(33 \%)$ & \\
\hline CRP (ever positive), n(\%) & & & & $<0.001$ \\
\hline Missing & $7(23 \%)$ & $23(8 \%)$ & $30(9 \%)$ & \\
\hline Negative & $13(57 \%)$ & $254(95 \%)$ & $267(92 \%)$ & \\
\hline Positive & $10(43 \%)$ & $14(5 \%)$ & $24(8 \%)$ & \\
\hline Received surfactant & $10(33 \%)$ & $16(5 \%)$ & $26(8 \%)$ & $<0.001$ \\
\hline Days on antibiotics & $9(2-14)$ & $4(2-7)$ & $4(2-7)$ & 0.006 \\
\hline WBC count (1st value) & $15.4(10.2-26.4)$ & $13.2(9.9-17)$ & $13.3(9.9-17.3)$ & 0.11 \\
\hline Missing, $\mathrm{n}(\%)$ & $2(7 \%)$ & $6(2 \%)$ & $8(2 \%)$ & \\
\hline Nasal canula & $24(80 \%)$ & $94(32 \%)$ & $118(37 \%)$ & $<0.001$ \\
\hline Nasal canula days & $2(1-5)$ & $1(1-2)$ & $1(1-2)$ & \\
\hline Oxyhood & $11(37 \%)$ & $182(63 \%)$ & $193(60 \%)$ & 0.010 \\
\hline Oxyhood days & $6(1.5-8)$ & $1(1-2)$ & $1(1-2)$ & \\
\hline Incubator & $6(20 \%)$ & $103(35 \%)$ & $109(34 \%)$ & 0.14 \\
\hline Incubator days & $3(2.25-3)$ & $1(1-2)$ & $1(1-2)$ & \\
\hline Delivery, $\mathrm{n}(\%)$ & & & & 0.38 \\
\hline Missing & $1(3 \%)$ & $2(1 \%)$ & $3(1 \%)$ & \\
\hline Vaginal & $12(41 \%)$ & $150(52 \%)$ & $162(51 \%)$ & \\
\hline Cesarean & $17(59 \%)$ & $139(48 \%)$ & $156(49 \%)$ & \\
\hline Gestational DM & $1(3 \%)$ & $21(7 \%)$ & $22(7 \%)$ & 0.67 \\
\hline PROM & $2(7 \%)$ & $20(7 \%)$ & $22(7 \%)$ & 0.99 \\
\hline Preeclampsia & $6(20 \%)$ & $25(9 \%)$ & $31(10 \%)$ & 0.091 \\
\hline
\end{tabular}

\subsection{Antibiotic Usage}

Excluding neonates who died during their stay, we characterized neonates based upon their duration of antibiotic treatment (1-7 days vs. $>7$ days, Table 2 ). 
Table 2. NICU characteristics by extended antibiotic use (excluding deaths)

\begin{tabular}{|c|c|c|c|c|}
\hline & $\begin{array}{l}>7 \text { Days } \\
(n=61)\end{array}$ & $\begin{array}{l}\text { 1-7 Days } \\
(n=227)\end{array}$ & $\begin{array}{l}\text { Combined } \\
(\mathrm{n}=288)\end{array}$ & P-value \\
\hline Female, $\mathrm{n}(\%)$ & $31(51 \%)$ & $104(46 \%)$ & $135(48 \%)$ & 0.58 \\
\hline Gestational age, median (IQR) & $34(32-36)$ & $37(35-37)$ & $36(35-37)$ & $<0.001$ \\
\hline Missing, $n(\%)$ & $1(2 \%)$ & $3(1 \%)$ & $4(1 \%)$ & \\
\hline Days in hospital, median (IQR) & $14(10-24)$ & $3(2-5)$ & $4(3-8)$ & $<0.001$ \\
\hline \multicolumn{5}{|l|}{ Reason(s) for admission ${ }^{2}, \mathrm{n}(\%)$} \\
\hline Sepsis & $10(16 \%)$ & $15(7 \%)$ & $25(9 \%)$ & 0.031 \\
\hline RDS & $51(84 \%)$ & $137(60 \%)$ & $188(65 \%)$ & 0.001 \\
\hline Prematurity & $44(72 \%)$ & $101(44 \%)$ & $145(50 \%)$ & $<0.001$ \\
\hline Heart Disease & $1(2 \%)$ & $2(1 \%)$ & $3(1 \%)$ & 0.99 \\
\hline Congenital Malformation & $4(7 \%)$ & $10(4 \%)$ & $14(5 \%)$ & 0.72 \\
\hline Overweight Infant & $0(0 \%)$ & $17(7 \%)$ & $17(6 \%)$ & 0.058 \\
\hline Low birth weight infant & $11(18 \%)$ & $29(13 \%)$ & $40(14 \%)$ & 0.40 \\
\hline Jaundice & $5(8 \%)$ & $11(5 \%)$ & $16(5 \%)$ & 0.48 \\
\hline Low Apgar score & $6(10 \%)$ & $8(4 \%)$ & $14(5 \%)$ & 0.089 \\
\hline Seizures & $0(0 \%)$ & $1(<1 \%)$ & $1(<1 \%)$ & 0.99 \\
\hline Hypoglycemia & $0(0 \%)$ & $4(2 \%)$ & $4(1 \%)$ & 0.67 \\
\hline Neonatal Asphyxia & $5(8 \%)$ & $5(2 \%)$ & $10(4 \%)$ & 0.061 \\
\hline Other & $16(26 \%)$ & $77(34 \%)$ & $93(32 \%)$ & 0.32 \\
\hline Birth weight (kg) & $1.8325(1.7-2.213)$ & $2.5(2.15-3)$ & $2.3(2-2.9)$ & $<0.001$ \\
\hline Missing, $\mathrm{n}(\%)$ & $1(2 \%)$ & $0(0 \%)$ & $1(<1 \%)$ & \\
\hline Weight at 5 days $(\mathrm{kg})$ & $1.8(1.6-2.3)$ & $2.4(2.0-2.9)$ & $2.1(1.8-2.7)$ & $<0.001$ \\
\hline Missing, $\mathrm{n}(\%)$ & $13(21 \%)$ & $176(78 \%)$ & $189(66 \%)$ & \\
\hline Apgar at 1 minute & $6(4.3-7)$ & $7(6-7)$ & $7(6-7)$ & 0.001 \\
\hline Missing, $\mathrm{n}(\%)$ & $19(31 \%)$ & $115(51 \%)$ & $134(47 \%)$ & \\
\hline Apgar at 5 minutes & $8(6-8)$ & $8(7.75-8)$ & $8(7-8)$ & 0.007 \\
\hline Missing, $\mathrm{n}(\%)$ & $17(28 \%)$ & $95(42 \%)$ & $112(39 \%)$ & \\
\hline Vitamin D & $3.655(2.3-5.475)$ & $3.5(2.6-5)$ & $3.5(2.5-5)$ & 0.83 \\
\hline Missing, $n(\%)$ & $21(34 \%)$ & $75(33 \%)$ & $96(33 \%)$ & \\
\hline CRP (ever positive), $n(\%)$ & & & & 0.086 \\
\hline Missing & $3(5 \%)$ & $17(7 \%)$ & $20(7 \%)$ & \\
\hline Negative & $51(88 \%)$ & $200(95 \%)$ & $251(94 \%)$ & \\
\hline Positive & $7(12 \%)$ & $10(5 \%)$ & $17(6 \%)$ & \\
\hline Received surfactant & $11(18 \%)$ & $4(2 \%)$ & $15(5 \%)$ & $<0.001$ \\
\hline WBC count (1st value) & $10.8(8.4-16.8)$ & $13.3(10.3-17.1)$ & $13.2(9.9-17.1)$ & 0.046 \\
\hline Missing, $\mathrm{n}(\%)$ & $0(0 \%)$ & $6(3 \%)$ & $6(2 \%)$ & \\
\hline Vent & $5(8 \%)$ & $0(0 \%)$ & $5(2 \%)$ & $<0.001$ \\
\hline Vent days & $4(2-6)$ & - & $3(2-5.5)$ & \\
\hline Nasal canula & $39(64 \%)$ & $56(25 \%)$ & $95(33 \%)$ & $<0.001$ \\
\hline Nasal canula days & $1(1-43.75)$ & $1(1-1)$ & $1(1-2)$ & \\
\hline Oxyhood & $51(84 \%)$ & $130(57 \%)$ & $181(63 \%)$ & $<0.001$ \\
\hline Oxyhood days & $2(1-3.25)$ & $1(1-1)$ & $1(1-2)$ & \\
\hline Incubator & $34(56 \%)$ & $71(31 \%)$ & $105(37 \%)$ & $<0.001$ \\
\hline
\end{tabular}




\begin{tabular}{|c|c|c|c|c|}
\hline Incubator days & $2(1-3)$ & $1(1-2)$ & $1(1-2)$ & \\
\hline Delivery, n(\%) & & & & 0.035 \\
\hline Missing & $0(0 \%)$ & $2(1 \%)$ & $2(1 \%)$ & \\
\hline Vaginal & $24(39 \%)$ & $125(56 \%)$ & $149(52 \%)$ & \\
\hline Cesarean & $37(61 \%)$ & $100(44 \%)$ & 137 (48\%) & \\
\hline Gestational DM & $3(5 \%)$ & $18(8 \%)$ & $21(7 \%)$ & 0.60 \\
\hline PROM & $6(10 \%)$ & $13(6 \%)$ & $19(6 \%)$ & 0.39 \\
\hline Preeclampsia & $9(15 \%)$ & $16(7 \%)$ & $25(9 \%)$ & 0.10 \\
\hline
\end{tabular}

Percentages are computed using the number of infants with a non-missing value.

IQR: interquartile range

${ }^{1}$ To compare the distribution of patient characteristics by antibiotic use, we employ chi-square tests. Similarly, we use Wilcoxon rank sum tests for continuous variables by antibiotic use.

${ }^{2}$ Percentages may not sum to $100 \%$.

Factors that had a significant effect upon antibiotic use included lower gestational age, respiratory distress syndrome, prematurity, lower weight at birth and at five days, use of surfactant, and all methods of respiratory support $(\mathrm{p}<0.001)$. Negative binomial regression was utilized to determine independent predictors of length of antibiotic use (Table 3).

Table 3. Model Effects: Duration of Antibiotic Use

\begin{tabular}{|c|c|c|}
\hline & $\begin{array}{lll}\text { Incidence } & \text { Rate } & \text { Ratio } \\
(95 \% \text { CI }) & & \end{array}$ & P-value \\
\hline Gestational age (per 1 week) & $0.98(0.93,1.03)$ & 0.48 \\
\hline Admitted for sepsis & $1.46(1.09,1.95)$ & 0.010 \\
\hline Admitted for RDS & $1.12(0.91,1.37)$ & 0.28 \\
\hline Birth weight (3 vs. $2 \mathrm{~kg}$ ) & $0.57(0.48,0.67)$ & $<0.001$ \\
\hline Apgar at 1 minute (per 2 increase) & $0.80(0.70,0.91)$ & $<0.001$ \\
\hline Vitamin D (per 2.6 increase) & $1.09(0.99,1.21)$ & 0.067 \\
\hline PROM & $0.99(0.71,1.39)$ & 0.97 \\
\hline Preeclampsia & $1.30(0.99,1.72)$ & 0.058 \\
\hline Positive CRP & $1.48(1.02,2.15)$ & 0.040 \\
\hline
\end{tabular}

Admission for sepsis and positive CRP increase the expected number of days on antibiotics by some $46 \%$ and $48 \%$, respectively. Birthweight of $3 \mathrm{~kg}$ compared with $2 \mathrm{~kg}$ is associated with a $43 \%$ decrease in days on antibiotics (Incidence Rate Ratio [IRR]: 0.57; 95\% confidence interval [CI]: 0.48-0.67). A two-point increase in Apgar score at one minute is associated with a $20 \%$ reduction in antibiotic days (IRR: $0.80 ; 95 \%$ CI: $0.70-0.91$ ). We failed to detect any association between gestational age, respiratory distress syndrome admission, vitamin $\mathrm{D}$, PROM and preeclampsia with antibiotic use.

\subsection{Oxygen and Mechanical Ventilation}

Neonates who utilized oxygen compared to those who did not had a longer median hospital stay ( 5 vs. 3 days, $p$ $<0.001)$, more admissions for respiratory distress syndrome $(82 \%$ vs. $44 \%, \mathrm{p}<0.001)$, more prematurity $(61 \%$ vs. $38 \%, \mathrm{p}<0.001$ ), more days on antibiotics ( 4 vs. 3 days, $\mathrm{p}<0.001$ ), and were more likely to be placed in an incubator $(44 \%$ vs. $20 \%, \mathrm{p}<0.001)$ (Table 1$)$.

Neonates who utilized mechanical ventilation compared to those who did not had a lower median gestational age (31 vs. 36 weeks, $\mathrm{p}<0.001$ ), lower median birth weight (1.6 vs. $2.3 \mathrm{~kg}, \mathrm{p}<0.001$ ), lower Apgar scores at one and five minutes ( 4 and 6 vs. 7 and $8, p<0.001)$, more likely to receive surfactant (33\% vs. $5 \%, \mathrm{p}<0.001)$, and more likely to have been placed nasal canula $(80 \%$ vs. $32 \%$, p < 0.001$)$ (Table 1$)$. 


\subsection{Mortality}

Of 321 total infants admitted to the NICU, 28 infants $(8.7 \%)$ died prior to discharge. The most common admission diagnoses in infants who died were respiratory distress syndrome $(86 \%, \mathrm{p}=0.046)$ and prematurity $(75 \%, p=0.019)$. Comparing neonates who died in the NICU to those who survived, neonates who died had a lower gestational age (30 weeks vs. 36 weeks, $\mathrm{p}<0.001)$; lower median birth weight $(1.2 \mathrm{~kg}$ vs. $2.3 \mathrm{~kg}, \mathrm{p}<$ 0.001); and lower Apgar scores at one and five minutes (4 and 6 vs. 7 and $8, p<0.001$ ). Infants who died were more likely to have received surfactant than those who survived $(39 \%$ vs. $5 \%, \mathrm{p}<0.001)$, require mechanical ventilation $86 \%$ vs. $2 \%, \mathrm{p}<0.001)$ and more likely to have been placed on nasal canula $(71 \%$ v. $33 \%, p<0.001)$ (Table 1).

Adjusting for gestational age in separate logistic regression models, Apgar scores, use of mechanical ventilation or oxygen, and an elevated CRP level were associated with death at discharge. A two-point increase in Apgar at 1 minute was associated with a $74 \%$ decrease in the odds of death (Odds Ratio [OR]: 0.26 ; 95\% CI: 0.12-0.57). A one-point increase in Apgar at 5 minutes was associated with a 44\% decrease in the odds of death (OR: 0.56; 95\% CI: 0.37-0.83). Elevated CRP level was associated with a 10.5 time higher odds of death (OR: 10.52; $95 \%$ CI: 2.25-49.2). No significant associations were detected for gender, sepsis, respiratory distress syndrome, nasal canula, birth weight, maternal preeclampsia, method of delivery, and PROM with neonatal mortality (Table 4).

Table 4. Model Effects: Odds of Death at Discharge (adjusted for gestational age)

\begin{tabular}{llll}
\hline & N & Odds Ratio $\mathbf{~ ( 9 5 \% ~ C I ) ~}$ & P-value \\
\hline Female & 317 & $1.80(0.63,5.14)$ & 0.27 \\
Admitted for sepsis & 317 & $1.10(0.24,4.97)$ & 0.90 \\
Admitted for RDS & 317 & $0.66(0.15,2.84)$ & 0.57 \\
Birth weight (3 vs. 2 kg) & 316 & $0.44(0.17,1.09)$ & 0.11 \\
Apgar at 1 minute (per 2 increase) & 178 & $0.26(0.12,0.57)$ & $<0.001$ \\
Apgar at 5 minutes (per 1 increase) & 201 & $0.56(0.37,0.83)$ & 0.004 \\
Days on antibiotics (per 5 days) & 312 & $0.73(0.48,1.13)$ & 0.16 \\
PROM & 308 & $0.69(0.10,4.71)$ & 0.71 \\
Preeclampsia & 308 & $2.41(0.61,9.48)$ & 0.21 \\
Positive CRP & 288 & $10.52(2.25,49.16)$ & 0.003 \\
C-section (vs. vaginal) & 314 & $0.51(0.17,1.57)$ & 0.24 \\
Received mechanical ventalation & 316 & $776.00(57.50,10473.00)$ & $<0.001$ \\
Received oxyhood & 314 & $0.26(0.08,0.78)$ & 0.017 \\
Received nasal canula & 314 & $2.40(0.74,7.80)$ & 0.15 \\
\hline
\end{tabular}

${ }^{1}$ Adjusted for gestational age at birth.

\section{Discussion}

In a prospective cohort study at the Al-Bashir NICU in Amman, Jordan, the most common reasons for admission were respiratory distress syndrome and prematurity, and their median gestational age would be considered late pre-term infants ( $>36$ weeks). Our cohort had an overall mortality of $8.7 \%$ and those neonates who died had a median gestational age of 30 weeks. Neonatal morbidity has grown to represent a larger proportion of overall infant mortality during the last two decades in Jordan, with prematurity as the leading cause of the mortality rate of children under five years of age (34\%), followed by congenital anomalies $(21 \%)$ and birth asphyxia (12\%) (Country, 2008-2013; WHO, 2013; Khoury \& Mas'ad, 2002). This is compatible with global data, with $40 \%$ of deaths in children younger than five years occurring in the neonatal period, and $99 \%$ of neonatal deaths occurring in low- or middle-income countries (Lawn, Wilczynska-Ketenda, \& Cousens, 2006).

The 2010 World Health Organization (WHO) data regarding neonatal mortality indicate that 989 of 1,975 (50\%) neonates aged 0 to 27 days are due to complications from prematurity. Our study is consistent with these findings, as half of the neonates admitted to the NICU were premature with a higher percentage of premature neonates 
requiring oxygen. Moreover, neonates that required mechanical ventilation or died had even lower gestational ages than those who did not require mechanical ventilation or those who survived. Since nearly $15 \%$ of all childhood deaths under age five are caused by prematurity-related complications, medical management of prematurity constitutes a large part of reducing childhood mortality and must be emphasized in developing nations such as Jordan with high rates of prematurity (WHO, 2013).

Current data pertaining to NICU outcomes in the United States has established that neonates admitted with gestational ages of 26 weeks have mortality rates as low as 9.7\% (Sparks, Caughey, \& Cheng, 2014). Infants with gestational ages of 22 to 26 weeks are often viable and can be considered for admission to the NICU (Sparks et al., 2014). However, although the median gestational age of infants admitted to this NICU in Jordan is 36 weeks, it is not always beneficial for resource-poor countries to admit extremely premature infants $(<28$ weeks). Extreme prematurity is associated with morbidities including retinopathy, patent ductus arteriosus, bronchopulmonary dysplasia, respiratory syncytial virus infection, interventricular hemorrhage, developmental delay, and necrotizing enterocolitis, which is a heavy financial burden for these resource-limited countries (Shapiro-Mendoza et al., 2008; Chang, 2011). In a Canadian study, cost per infant during the first ten years of life for gestational ages less than 28 weeks was $\$ 67,467$ versus $\$ 10,010$ for infants born at 33-36 weeks due to such complications (Johnston, Gooch, Korol, Vo, Eyawo, Bradt, \& Levy 2014). In the United States, cost for term infants is around $\$ 2,061$ versus $\$ 26,054$ for late-preterm infants, and costs within the first year of life were three times higher for late-preterm infants (McLaurin, Hall, Jackson, Owens, \& Mahadevia, 2009). While cost analyses have not been conducted in Jordan, it is difficult to appropriate funds in resource-limited nations to sufficiently treat certain severe prematurity-associated complications (Johnston et al., 2014; Abu-Salah, 2011). However, other prematurity-associated complications such as retinopathy of prematurity can be screened for in the neonatal setting within a developing nation and treated early in a cost-effective manner (Zin, Magluta, Pinto, Entringer, Mendes-Gomes, Moreira, \& Gilbert, 2014). Furthermore, some complications such as low birth weight can potentially be prevented with improved prenatal maternal follow-up and education (Dai, Mao, Luo, \& Shen, 2014). Therefore, in order to diminish neonatal mortality in such nations, organizations such as the WHO must consider financial aspects of treatment.

Despite the high financial burden of caring for extremely premature infants in a developing nation, there are preliminary steps that can be taken to decrease current costs of neonatal healthcare. One aspect of improvement includes the current lack of universal screening for group B Streptococcus (GBS) in Jordan. As a result, GBS status in mothers is unknown and likely contributes to a significant percentage of the cases of sepsis and respiratory distress syndrome from this cohort. Provision of routine screening for infections such as GBS and CMV along with prophylactic treatment would be a cost-effective method to diminish subsequent costs of treatment and extended hospital stays (Alkhawaja, Ismaeel, Botta, \& Senok, 2012). A second area of improvement of neonatal health in this study includes respiratory distress syndrome (RDS), which was a key exposure for mortality, extended antibiotic use, and requiring oxygen. This high rate of RDS could be due to preventable environmental exposures such as the high rates of smoking, including Amman (Khuri-Bulos et al., 2013). Environmental exposures could also be a reason for the low median birth weight found in this cohort (2.3 $\mathrm{kg}$ ), which was a significant factor for extended antibiotic use, mechanical ventilation, and mortality.

This study is the first to provide comprehensive information regarding outcomes of neonates in a representative cohort in the Middle Eastern region. Although this study is the first to describe neonatal outcomes in Amman, Jordan, there are certain limitations. Chart abstraction was acquired through paper records, with missing records, which could potentially alter statistics such as mortality rate and life-threatening prematurity-related complications such as interventricular hemorrhage. Furthermore, due to suboptimal microbiological conditions, not all neonates had documented infections. Our results indicate that efforts must be made in order to improve treatment of neonates currently admitted to the NICU and prevent neonatal morbidities such as RDS and deaths that we know to be avoidable per data from developed nations. Pertinent variables include maternal prenatal and perinatal care, and medication and equipment used in the NICU. Also, we must determine preventable environmental causes of neonatal respiratory distress syndrome and prematurity and also evaluate how to fund excess costs incurred by the treatment of extremely premature infants. These efforts will help meet the United Nations' goal of a reduction of neonatal mortality. Of note, neonatal medicine has been introduced to Middle Eastern nations within the past two decades, while neonatal medicine was established in the United States by 1973. Therefore, the current discrepancies in neonatal care could also be attributed to the relative novelty of this field of care and resources in nations such as Jordan (Rezaeizadeh, Nayeri, \& Shariat, 2014).

\section{Conclusion}

In a retrospective analysis of Middle Eastern NICU outcomes, infants admitted to the Jordanian NICU have 
significantly higher median gestational age and birth weights than in developed countries. Among this population, low Apgar scores, use of mechanical ventilation or oxygen, and positive CRP were associated with mortality. Further data collection is necessary in order to increase the power of this study and follow-up studies are necessary in order to work towards the current United Nations Millennium Development Goal of reducing childhood mortality. Longitudinal studies are needed to characterize subsequent outcomes that are not measured in this study.

\section{Acknowledgements}

We would like to thank the clinical research staff at Al-Basheer Hospital in Amman, Jordan for their assistance with their project. All authors decline competing interests. Funding was provided by the Vanderbilt Medical School Emphasis Research Program and Overall Global Health Fellowship.

\section{References}

Abu-Salah, O. (2011). Unfavorable outcomes associated with late preterm birth: observations from Jordan. Journal of Pakistan Medical Association, 61-64.

Alkhawaja, S., Ismaeel, A., Botta, G., \& Senok, A. C. (2012). The prevalence of congenital and perinatal cytomegalovirus infections among newborns of seropositive mothers. The Journal of Infection in Developing Countries, 6, 410-415.

Chang, L. W. (2011). Incidence and risk factors of bronchopulmonary dysplasia in premature infants in 10 hospitals in China. Collaborative Study Group for Bronchopulmonary Dysplasia of Prematurity in China, 49, 655-662.

Country Cooperation Strategy for WHO and Jordan 2008-2013. (2009). W.R.O.f.t.E. Mediterranean, Editor. Retrieved from http://www.who.int/countryfocus/cooperation_strategy/ccs_jor_en.pdf

Dai, L. L., Mao, Y. Y., Luo, X. M., \& Shen, Y. P. (2014). Prenatal care in combination with maternal education has a synergistic effect on the risk of neonatal low birth weight: new findings in a retrospective cohort city in Kunshan City, China. Plos One, 9(11).

Feng, Y. (2011). Causes of death in neonatal intensive care units. Australian Medical Student Journal, 41-43.

Harris, P. A., Taylor, R., Thielke, R., Payne, J., Gonzalez, N., \& Conde, J. G. (2009). Research electronic data capture (REDCap)--a metadata-driven methodology and workflow process for providing translational research informatics support. Journal of Biomedical Information, 42, 377-381.

Johnston, K. M., Gooch, K., Korol, E., Vo, P., Eyawo, O., Bradt, P., \& Levy, A. (2014). The economic burden of prematurity in Canada. BMC Pediatrics, 14. http://dx.doi.org/10.1186/1471-2431-14-93

Khoury, S., \& Mas'ad, D. (2002). Causes of Infant Mortality in Jordan. Saudi Medical Journal, 23, 432-435.

Khuri-Bulos, N., Lang, R. D., Blevins, M., Kudyba, K., Lawrence, L., Davidson, M., ... Halasa, N. (2013). Vitamin D deficiency among newborns in Amman, Jordan. Global Journal of Health Science, 6, 162-171.

Lawn, J. E., Wilczynska-Ketenda, K., \& Cousens, S. N. (2006). Estimating the causes of 4 million neonatal deaths in the year 2000. International Journal of Epidemiology, 35, 706-718.

McLaurin, K. K., Hall, C. B., Jackson, E. A., Owens, O. V., \& Mahadevia, P. V. (2009). Perisistence of Morbidity and cost differences between late-preterm and term infants during the first year of life. Pediatrics, 123, 653-659.

Rezaeizadeh, G., Nayeri, F., \& Shariat, M. (2014). A history of neonatal medicine in Iran. Archives of Iranian Medicine, 17(12), 855-861.

Shapiro-Mendoza, C. K., Tomashek, K. M., Kotelchuck, M., Barfield, W., Nannini, A., Weiss, J., \& Declercq, E. (2008). Effect of late-preterm birth and maternal medical conditions on newborn morbidity risk. Pediatrics, $121,223-232$.

Simpson, C. D. A., Xiang, Y. Y., Hellmann, J., \& Tomlinson, C. (2010). Trends in cause-specific mortality at a Canadian outborn NICU. Pediatrics, 126, 1538-1544.

Sparks, T., Caughey, A., \& Cheng, Y. (2014). Neonatal outcomes at periviable gestaional ages: does half a week make a difference? American Journal of Obstetrics and Gynecology, 210, 108.

United Nations. (2010). The millennium development goals report 2010. New York: United Nations. Retrieved from http://www.un.org/millenniumgoals/ 
World Health Organization. (2013). Jordan Health Profile. Retrieved from http://www.who.int/gho/countries/jor.pdf.

Zin, A. A., Magluta, C., Pinto, M. F., Entringer, A. P., Mendes-Gomes, M. A., Moreira, M. E., \& Gilbert, C. (2014). Retinopathy of prematurity screening and treatment cost in Brazil. Revista Panamerica De Salud Publica, 36(1), 37-43.

\section{Appendix A}

\section{List of Tables Included in Manuscript}

Table 1A. NICU Characteristics by Vital Status at Discharge, Oxyhood, and Ventilator Use

Vital Status

\begin{tabular}{|c|c|c|c|c|}
\hline & $\begin{array}{l}\text { Dead } \\
(n=28)\end{array}$ & $\begin{array}{l}\text { Alive } \\
(n=293)\end{array}$ & $\begin{array}{l}\text { Combined } \\
(n=321)\end{array}$ & P-value \\
\hline Female & $14(50 \%)$ & $140(48 \%)$ & $154(48 \%)$ & 0.98 \\
\hline Gestational age & $30(29-33)$ & $36(35-37)$ & $36(34-37)$ & $<0.001$ \\
\hline Missing, $\mathrm{n}(\%)$ & $0(0 \%)$ & $4(1 \%)$ & $4(1 \%)$ & \\
\hline Days in hospital & $6(2-11)$ & $4(3-8)$ & $4(2-8)$ & 0.65 \\
\hline \multicolumn{5}{|l|}{ Reason(s) for admission } \\
\hline Sepsis & $6(21 \%)$ & $25(9 \%)$ & $31(10 \%)$ & 0.061 \\
\hline RDS & $24(86 \%)$ & $191(65 \%)$ & $215(67 \%)$ & 0.046 \\
\hline Neonatal pneumonia & $1(4 \%)$ & $0(0 \%)$ & $1(<1 \%)$ & 0.14 \\
\hline Prematurity & $21(75 \%)$ & $146(50 \%)$ & $167(52 \%)$ & 0.019 \\
\hline Heart Disease & $2(7 \%)$ & $4(1 \%)$ & $6(2 \%)$ & 0.15 \\
\hline Congenital Malformation & $4(14 \%)$ & $14(5 \%)$ & $18(6 \%)$ & 0.097 \\
\hline Overweight Infant & $0(0 \%)$ & $17(6 \%)$ & $17(5 \%)$ & 0.39 \\
\hline Low birth weight infant & $3(11 \%)$ & $42(14 \%)$ & $45(14 \%)$ & 0.81 \\
\hline Jaundice & $0(0 \%)$ & $16(5 \%)$ & $16(5 \%)$ & 0.42 \\
\hline Low Apgar score & $4(14 \%)$ & $15(5 \%)$ & $19(6 \%)$ & 0.12 \\
\hline Seizures & $0(0 \%)$ & $1(<1 \%)$ & $1(<1 \%)$ & 0.99 \\
\hline Hypoglycemia & $0(0 \%)$ & $4(1 \%)$ & $4(1 \%)$ & 0.99 \\
\hline Neonatal Asphyxia & $3(11 \%)$ & $11(4 \%)$ & $14(4 \%)$ & 0.22 \\
\hline Other & $5(18 \%)$ & $94(32 \%)$ & $99(31 \%)$ & 0.18 \\
\hline Birth weight (kg) & $1.2(0.9-1.9)$ & $2.3(2-2.9)$ & $2.3(1.9-2.9)$ & $<0.001$ \\
\hline Missing, $\mathrm{n}(\%)$ & $0(0 \%)$ & $1(<1 \%)$ & $1(<1 \%)$ & \\
\hline Weight at 5 days $(\mathrm{kg})$ & $1.3(1.2-1.6)$ & $\begin{array}{l}2.07(1.785- \\
2.65)\end{array}$ & $2(1.73-2.6)$ & $<0.001$ \\
\hline Missing, $\mathrm{n}(\%)$ & $19(68 \%)$ & $194(66 \%)$ & $213(66 \%)$ & \\
\hline Apgar at 1 minute & $4(3-5)$ & $7(6-7)$ & $7(5-7)$ & $<0.001$ \\
\hline Missing, $n(\%)$ & $7(25 \%)$ & $135(46 \%)$ & $142(44 \%)$ & \\
\hline Apgar at 5 minutes & $6(5-7.5)$ & $8(7-8)$ & $8(7-8)$ & $<0.001$ \\
\hline Missing, $\mathrm{n}(\%)$ & $5(18 \%)$ & $113(39 \%)$ & $118(37 \%)$ & \\
\hline CRP (ever positive), n(\%) & & & & $<0.001$ \\
\hline Missing & $9(32 \%)$ & $21(7 \%)$ & $30(9 \%)$ & \\
\hline Negative & $12(63 \%)$ & $255(94 \%)$ & $267(92 \%)$ & \\
\hline
\end{tabular}




\begin{tabular}{|c|c|c|c|c|}
\hline Positive & $7(37 \%)$ & $17(6 \%)$ & $24(8 \%)$ & \\
\hline Received surfactant & $11(39 \%)$ & $15(5 \%)$ & $26(8 \%)$ & $<0.001$ \\
\hline Days on antibiotics & $6(2-10)$ & $4(2-7)$ & $4(2-7)$ & 0.44 \\
\hline WBC count (1st value) & $15.4(9.7-25.4)$ & $\begin{array}{l}13.2(9.9- \\
17.1)\end{array}$ & $\begin{array}{l}13.3(9.9- \\
17.3)\end{array}$ & 0.21 \\
\hline Missing, $\mathrm{n}(\%)$ & $2(7 \%)$ & $6(2 \%)$ & $8(2 \%)$ & \\
\hline Vent & $24(86 \%)$ & $6(2 \%)$ & $30(9 \%)$ & $<0.001$ \\
\hline Vent days & $2.5(1-6.25)$ & $3(2-5.5)$ & $2.5(1-6)$ & \\
\hline Nasal canula & $20(71 \%)$ & $98(33 \%)$ & $118(37 \%)$ & $<0.001$ \\
\hline Nasal canula days & $2(1.5-4.5)$ & $1(1-2)$ & $1(1-2)$ & \\
\hline Oxyhood & $9(32 \%)$ & $184(63 \%)$ & $193(60 \%)$ & 0.003 \\
\hline Oxyhood days & $6(1-8)$ & $1(1-2)$ & $1(1-2)$ & \\
\hline Incubator & $2(7 \%)$ & $107(37 \%)$ & $109(34 \%)$ & 0.003 \\
\hline Incubator days & $6(3-8)$ & $1(1-2)$ & $1(1-2)$ & \\
\hline Delivery, $\mathrm{n}(\%)$ & & & & 0.61 \\
\hline Missing & $1(4 \%)$ & $2(1 \%)$ & $3(1 \%)$ & \\
\hline Vaginal & $12(44 \%)$ & $150(52 \%)$ & $162(51 \%)$ & \\
\hline Cesarean & $15(56 \%)$ & $141(48 \%)$ & $156(49 \%)$ & \\
\hline Gestational DM & $1(4 \%)$ & $21(7 \%)$ & $22(7 \%)$ & 0.74 \\
\hline PROM & $3(11 \%)$ & $19(6 \%)$ & $22(7 \%)$ & 0.65 \\
\hline Preeclampsia & $5(18 \%)$ & $26(9 \%)$ & $31(10 \%)$ & 0.23 \\
\hline
\end{tabular}

Oxyhood use

\begin{tabular}{|c|c|c|c|c|}
\hline & $\begin{array}{l}\text { Oxyhood } \\
(n=193)\end{array}$ & $\begin{array}{l}\text { No Oxyhood } \\
(n=128)\end{array}$ & $\begin{array}{l}\text { Combined } \\
(n=321)\end{array}$ & P-value \\
\hline Female & $91(47 \%)$ & $63(49 \%)$ & $154(48 \%)$ & 0.80 \\
\hline Gestational age & $35(34-37)$ & $37(35-37)$ & $36(34-37)$ & 0.002 \\
\hline Missing, $\mathrm{n}(\%)$ & $0(0 \%)$ & $4(3 \%)$ & $4(1 \%)$ & \\
\hline Days in hospital & $5(3-9)$ & $3(2-6)$ & $4(2-8)$ & $<0.001$ \\
\hline \multicolumn{5}{|l|}{ Reason(s) for admission } \\
\hline Sepsis & $14(7 \%)$ & $17(13 \%)$ & $31(10 \%)$ & 0.11 \\
\hline RDS & $159(82 \%)$ & $56(44 \%)$ & $215(67 \%)$ & $<0.001$ \\
\hline Neonatal pneumonia & $0(0 \%)$ & $1(1 \%)$ & $1(<1 \%)$ & 0.84 \\
\hline Prematurity & $118(61 \%)$ & $49(38 \%)$ & $167(52 \%)$ & $<0.001$ \\
\hline Heart Disease & $2(1 \%)$ & $4(3 \%)$ & $6(2 \%)$ & 0.35 \\
\hline Congenital Malformation & $11(6 \%)$ & $7(5 \%)$ & $18(6 \%)$ & 0.99 \\
\hline Overweight Infant & $5(3 \%)$ & $12(9 \%)$ & $17(5 \%)$ & 0.016 \\
\hline Low birth weight infant & $27(14 \%)$ & $18(14 \%)$ & $45(14 \%)$ & 0.99 \\
\hline Jaundice & $8(4 \%)$ & $8(6 \%)$ & $16(5 \%)$ & 0.56 \\
\hline Low Apgar score & $12(6 \%)$ & $7(5 \%)$ & $19(6 \%)$ & 0.97 \\
\hline Seizures & $0(0 \%)$ & $1(1 \%)$ & $1(<1 \%)$ & 0.84 \\
\hline Hypoglycemia & $1(1 \%)$ & $3(2 \%)$ & $4(1 \%)$ & 0.35 \\
\hline Neonatal Asphyxia & $8(4 \%)$ & $6(5 \%)$ & $14(4 \%)$ & 0.99 \\
\hline
\end{tabular}




\begin{tabular}{|c|c|c|c|c|}
\hline Other & $44(23 \%)$ & $55(43 \%)$ & $99(31 \%)$ & $<0.001$ \\
\hline Death & $9(5 \%)$ & $19(15 \%)$ & $28(9 \%)$ & \\
\hline Birth weight (kg) & $2.2(1.9-2.8)$ & $2.5(2.1-3.1)$ & $2.29(1.9-2.9)$ & 0.005 \\
\hline Missing, $\mathrm{n}(\%)$ & $1(1 \%)$ & $0(0 \%)$ & $1(<1 \%)$ & \\
\hline Weight at 5 days (kg) & $1.9(1.8-2.5)$ & $2.36(1.665-2.95)$ & $2(1.7-2.6)$ & 0.32 \\
\hline Missing, $\mathrm{n}(\%)$ & $112(58 \%)$ & $101(79 \%)$ & $213(66 \%)$ & \\
\hline Apgar at 1 minute & $6(5-7)$ & $7(5-7)$ & $7(5-7)$ & 0.80 \\
\hline Missing, $\mathrm{n}(\%)$ & $79(41 \%)$ & $63(49 \%)$ & $142(44 \%)$ & \\
\hline Apgar at 5 minutes & $8(7-8)$ & $8(6-8)$ & $8(7-8)$ & 0.39 \\
\hline Missing, $\mathrm{n}(\%)$ & $66(34 \%)$ & $52(41 \%)$ & $118(37 \%)$ & \\
\hline Vitamin D & $3.5(2.5-4.8)$ & $3.5(2.5-5.3)$ & $3.5(2.5-5)$ & 0.63 \\
\hline Missing, $\mathrm{n}(\%)$ & $70(36 \%)$ & $37(29 \%)$ & $107(33 \%)$ & \\
\hline CRP (ever positive), $\mathrm{n}(\%)$ & & & & 0.82 \\
\hline Missing & $11(6 \%)$ & $19(15 \%)$ & $30(9 \%)$ & \\
\hline Negative & $168(92 \%)$ & $99(91 \%)$ & $267(92 \%)$ & \\
\hline Positive & $14(8 \%)$ & $10(9 \%)$ & $24(8 \%)$ & \\
\hline Received surfactant & $17(9 \%)$ & $9(7 \%)$ & $26(8 \%)$ & 0.717 \\
\hline Days on antibiotics & $4(3-8)$ & $3(2-5)$ & $4(2-7)$ & $<0.001$ \\
\hline WBC count (1st value) & $12.3(9.7-16.5)$ & $15(10.5-18.9)$ & $13.3(9.9-17.3)$ & 0.003 \\
\hline Missing, $\mathrm{n}(\%)$ & $4(2 \%)$ & $4(3 \%)$ & $8(2 \%)$ & \\
\hline Vent & $11(6 \%)$ & $19(15 \%)$ & $30(9 \%)$ & 0.010 \\
\hline Vent days & $3(1.5-5)$ & $2(1-6.5)$ & $2.5(1-6)$ & \\
\hline Nasal canula & $67(35 \%)$ & $51(40 \%)$ & $118(37 \%)$ & 0.42 \\
\hline Nasal canula days & $1(1-2.8)$ & $1(1-2)$ & $1(1-2)$ & \\
\hline Incubator & $84(44 \%)$ & $25(20 \%)$ & $109(34 \%)$ & $<0.001$ \\
\hline Incubator days & $2(1-2)$ & $1(1-2)$ & $1(1-2)$ & \\
\hline Delivery, n(\%) & & & & 0.99 \\
\hline Missing & $2(1 \%)$ & $1(1 \%)$ & $3(1 \%)$ & \\
\hline Vaginal & $97(51 \%)$ & $65(51 \%)$ & $162(51 \%)$ & \\
\hline Cesarean & $94(49 \%)$ & $62(49 \%)$ & $156(49 \%)$ & \\
\hline Gestational DM & $9(5 \%)$ & $13(10 \%)$ & $22(7 \%)$ & 0.093 \\
\hline PROM & $11(6 \%)$ & $11(9 \%)$ & $22(7 \%)$ & 0.44 \\
\hline Preeclampsia & $19(10 \%)$ & $12(9 \%)$ & $31(10 \%)$ & 0.99 \\
\hline
\end{tabular}

Mechanical Ventilation

\begin{tabular}{lllll}
\hline & $\begin{array}{l}\text { Ventilator } \\
(\mathbf{n = 3 0 )}\end{array}$ & $\begin{array}{l}\text { No Ventilator } \\
(\mathbf{n = 2 9 1 )}\end{array}$ & $\begin{array}{l}\text { Combined } \\
(\mathbf{n = 3 2 1})\end{array}$ & P-value \\
\hline Female & $17(57 \%)$ & $137(47 \%)$ & $154(48 \%)$ & 0.42 \\
Gestational age & $31(29.3-354.8)$ & $36(35-37)$ & $36(34-37)$ & $<0.001$ \\
Missing, n(\%) & $0(0 \%)$ & $4(1 \%)$ & $4(1 \%)$ & 0.012 \\
$\begin{array}{l}\text { Days in hospital } \\
\text { Reason(s) for admission }\end{array}$ & $109.5(3-14.8)$ & $4(2-8)$ & $4(2-8)$ & 0.091
\end{tabular}




\begin{tabular}{|c|c|c|c|c|}
\hline RDS & $25(83 \%)$ & $190(65 \%)$ & $215(67 \%)$ & 0.072 \\
\hline Neonatal pneumonia & $1(3 \%)$ & $0(0 \%)$ & $1(<1 \%)$ & 0.16 \\
\hline Prematurity & $21(70 \%)$ & $146(50 \%)$ & $167(52 \%)$ & 0.060 \\
\hline Heart Disease & $3(10 \%)$ & $3(1 \%)$ & $6(2 \%)$ & 0.006 \\
\hline Congenital Malformation & $3(10 \%)$ & $15(5 \%)$ & $18(6 \%)$ & 0.50 \\
\hline Overweight Infant & $0(0 \%)$ & $17(6 \%)$ & $17(5 \%)$ & 0.35 \\
\hline Low birth weight infant & $4(13 \%)$ & $41(14 \%)$ & $45(14 \%)$ & 0.99 \\
\hline Jaundice & $0(0 \%)$ & $16(5 \%)$ & $16(5 \%)$ & 0.38 \\
\hline Low Apgar score & $5(17 \%)$ & $14(5 \%)$ & $19(6 \%)$ & 0.027 \\
\hline Seizures & $0(0 \%)$ & $1(<1 \%)$ & $1(<1 \%)$ & 0.99 \\
\hline Hypoglycemia & $0(0 \%)$ & $4(1 \%)$ & $4(1 \%)$ & 0.99 \\
\hline Neonatal Asphyxia & $4(13 \%)$ & $10(3 \%)$ & $14(4 \%)$ & 0.040 \\
\hline Other & $7(23 \%)$ & $92(32 \%)$ & $99(31 \%)$ & 0.47 \\
\hline Death & $24(80 \%)$ & $4(1 \%)$ & $28(9 \%)$ & \\
\hline Birth weight (kg) & $1.55(1-2)$ & $2.3(2-2.9)$ & $2.29(1.9-2.9)$ & $<0.001$ \\
\hline Missing, $\mathrm{n}(\%)$ & $0(0 \%)$ & $1(<1 \%)$ & $1(<1 \%)$ & \\
\hline Weight at 5 days $(\mathrm{kg})$ & $1.6(1.2-1.9)$ & $2.07(1.77-2.63)$ & $2(1.73-2.6)$ & 0.006 \\
\hline Missing, $n(\%)$ & $17(57 \%)$ & $196(67 \%)$ & $213(66 \%)$ & \\
\hline Apgar at 1 minute & $4(2.75-5)$ & $7(6-7)$ & $7(5-7)$ & $<0.001$ \\
\hline Missing, $\mathrm{n}(\%)$ & $6(20 \%)$ & $136(47 \%)$ & $142(44 \%)$ & \\
\hline Apgar at 5 minutes & $6(5-7)$ & $8(7-8)$ & $8(7-8)$ & $<0.001$ \\
\hline Missing, $\mathrm{n}(\%)$ & $5(17 \%)$ & $113(39 \%)$ & $118(37 \%)$ & \\
\hline Vitamin D & $\begin{array}{l}3.7(2.985- \\
5.765)\end{array}$ & $3.5(2.5-5)$ & $3.5(2.5-5)$ & 0.48 \\
\hline Missing, $\mathrm{n}(\%)$ & $11(37 \%)$ & $96(33 \%)$ & $107(33 \%)$ & \\
\hline CRP (ever positive), $\mathrm{n}(\%)$ & & & & $<0.001$ \\
\hline Missing & $7(23 \%)$ & $23(8 \%)$ & $30(9 \%)$ & \\
\hline Negative & $13(57 \%)$ & $254(95 \%)$ & $267(92 \%)$ & \\
\hline Positive & $10(43 \%)$ & $14(5 \%)$ & $24(8 \%)$ & \\
\hline Received surfactant & $10(33 \%)$ & $16(5 \%)$ & $26(8 \%)$ & $<0.001$ \\
\hline Days on antibiotics & $9(2-14)$ & $4(2-7)$ & $4(2-7)$ & 0.006 \\
\hline WBC count (1st value) & $15.4(10.2-26.4)$ & $13.2(9.9-17)$ & $13.3(9.9-17.3)$ & 0.11 \\
\hline Missing, $\mathrm{n}(\%)$ & $2(7 \%)$ & $6(2 \%)$ & $8(2 \%)$ & \\
\hline Nasal canula & $24(80 \%)$ & $94(32 \%)$ & $118(37 \%)$ & $<0.001$ \\
\hline Nasal canula days & $2(1-5)$ & $1(1-2)$ & $1(1-2)$ & \\
\hline Oxyhood & $11(37 \%)$ & $182(63 \%)$ & $193(60 \%)$ & 0.010 \\
\hline Oxyhood days & $6(1.5-8)$ & $1(1-2)$ & $1(1-2)$ & \\
\hline Incubator & $6(20 \%)$ & $103(35 \%)$ & $109(34 \%)$ & 0.14 \\
\hline Incubator days & $3(2.25-3)$ & $1(1-2)$ & $1(1-2)$ & \\
\hline Delivery, n(\%) & & & & 0.38 \\
\hline Missing & $1(3 \%)$ & $2(1 \%)$ & $3(1 \%)$ & \\
\hline Vaginal & $12(41 \%)$ & $150(52 \%)$ & $162(51 \%)$ & \\
\hline Cesarean & $17(59 \%)$ & $139(48 \%)$ & $156(49 \%)$ & \\
\hline
\end{tabular}




\begin{tabular}{lllll} 
Gestational DM & $1(3 \%)$ & $21(7 \%)$ & $22(7 \%)$ & 0.67 \\
PROM & $2(7 \%)$ & $20(7 \%)$ & $22(7 \%)$ & 0.99 \\
Preeclampsia & $6(20 \%)$ & $25(9 \%)$ & $31(10 \%)$ & 0.091 \\
\hline
\end{tabular}

Table 1B. NICU characteristics by extended antibiotic use (excluding deaths)

\begin{tabular}{|c|c|c|c|c|}
\hline & $\begin{array}{l}>7 \text { Days } \\
(n=61)\end{array}$ & $\begin{array}{l}\text { 1-7 Days } \\
(n=227)\end{array}$ & $\begin{array}{l}\text { Combined } \\
(n=288)\end{array}$ & P-value \\
\hline Female, $\mathrm{n}(\%)$ & $31(51 \%)$ & $104(46 \%)$ & $135(48 \%)$ & 0.58 \\
\hline Gestational age, median (IQR) & $34(32-36)$ & $37(35-37)$ & $36(35-37)$ & $<0.001$ \\
\hline Missing, $\mathrm{n}(\%)$ & $1(2 \%)$ & $3(1 \%)$ & $4(1 \%)$ & \\
\hline Days in hospital, median (IQR) & $14(10-24)$ & $3(2-5)$ & $4(3-8)$ & $<0.001$ \\
\hline \multicolumn{5}{|l|}{ Reason(s) for admission ${ }^{2}, \mathrm{n}(\%)$} \\
\hline Sepsis & $10(16 \%)$ & $15(7 \%)$ & $25(9 \%)$ & 0.031 \\
\hline RDS & $51(84 \%)$ & $137(60 \%)$ & $188(65 \%)$ & 0.001 \\
\hline Prematurity & $44(72 \%)$ & $101(44 \%)$ & $145(50 \%)$ & $<0.001$ \\
\hline Heart Disease & $1(2 \%)$ & $2(1 \%)$ & $3(1 \%)$ & 0.99 \\
\hline Congenital Malformation & $4(7 \%)$ & $10(4 \%)$ & $14(5 \%)$ & 0.72 \\
\hline Overweight Infant & $0(0 \%)$ & $17(7 \%)$ & $17(6 \%)$ & 0.058 \\
\hline Low birth weight infant & $11(18 \%)$ & $29(13 \%)$ & $40(14 \%)$ & 0.40 \\
\hline Jaundice & $5(8 \%)$ & $11(5 \%)$ & $16(5 \%)$ & 0.48 \\
\hline Low Apgar score & $6(10 \%)$ & $8(4 \%)$ & $14(5 \%)$ & 0.089 \\
\hline Seizures & $0(0 \%)$ & $1(<1 \%)$ & $1(<1 \%)$ & 0.99 \\
\hline Hypoglycemia & $0(0 \%)$ & $4(2 \%)$ & $4(1 \%)$ & 0.67 \\
\hline Neonatal Asphyxia & $5(8 \%)$ & $5(2 \%)$ & $10(4 \%)$ & 0.061 \\
\hline Other & $16(26 \%)$ & $77(34 \%)$ & $93(32 \%)$ & 0.32 \\
\hline Birth weight (kg) & $1.8325(1.7-2.213)$ & $2.5(2.15-3)$ & $2.3(2-2.9)$ & $<0.001$ \\
\hline Missing, $\mathrm{n}(\%)$ & $1(2 \%)$ & $0(0 \%)$ & $1(<1 \%)$ & \\
\hline Weight at 5 days $(\mathrm{kg})$ & $1.8(1.6-2.3)$ & $2.4(2.0-2.9)$ & $2.1(1.8-2.7)$ & $<0.001$ \\
\hline Missing, $\mathrm{n}(\%)$ & $13(21 \%)$ & $176(78 \%)$ & $189(66 \%)$ & \\
\hline Apgar at 1 minute & $6(4.3-7)$ & $7(6-7)$ & $7(6-7)$ & 0.001 \\
\hline Missing, $\mathrm{n}(\%)$ & $19(31 \%)$ & $115(51 \%)$ & $134(47 \%)$ & \\
\hline Apgar at 5 minutes & $8(6-8)$ & $8(7.75-8)$ & $8(7-8)$ & 0.007 \\
\hline Missing, $\mathrm{n}(\%)$ & $17(28 \%)$ & $95(42 \%)$ & $112(39 \%)$ & \\
\hline Vitamin D & $3.655(2.3-5.475)$ & $3.5(2.6-5)$ & $3.5(2.5-5)$ & 0.83 \\
\hline Missing, $\mathrm{n}(\%)$ & $21(34 \%)$ & $75(33 \%)$ & $96(33 \%)$ & \\
\hline CRP (ever positive), n(\%) & & & & 0.086 \\
\hline Missing & $3(5 \%)$ & $17(7 \%)$ & $20(7 \%)$ & \\
\hline Negative & $51(88 \%)$ & $200(95 \%)$ & $251(94 \%)$ & \\
\hline Positive & $7(12 \%)$ & $10(5 \%)$ & $17(6 \%)$ & \\
\hline Received surfactant & $11(18 \%)$ & $4(2 \%)$ & $15(5 \%)$ & $<0.001$ \\
\hline WBC count (1st value) & $10.8(8.4-16.8)$ & $13.3(10.3-17.1)$ & $13.2(9.9-17.1)$ & 0.046 \\
\hline Missing, $\mathrm{n}(\%)$ & $0(0 \%)$ & $6(3 \%)$ & $6(2 \%)$ & \\
\hline Vent & $5(8 \%)$ & $0(0 \%)$ & $5(2 \%)$ & $<0.001$ \\
\hline
\end{tabular}




\begin{tabular}{|c|c|c|c|c|}
\hline Vent days & $4(2-6)$ & - & $3(2-5.5)$ & \\
\hline Nasal canula & $39(64 \%)$ & $56(25 \%)$ & $95(33 \%)$ & $<0.001$ \\
\hline Nasal canula days & $1(1-43.75)$ & $1(1-1)$ & $1(1-2)$ & \\
\hline Oxyhood & $51(84 \%)$ & $130(57 \%)$ & $181(63 \%)$ & $<0.001$ \\
\hline Oxyhood days & $2(1-3.25)$ & $1(1-1)$ & $1(1-2)$ & \\
\hline Incubator & $34(56 \%)$ & $71(31 \%)$ & $105(37 \%)$ & $<0.001$ \\
\hline Incubator days & $2(1-3)$ & $1(1-2)$ & $1(1-2)$ & \\
\hline Delivery, n(\%) & & & & 0.035 \\
\hline Missing & $0(0 \%)$ & $2(1 \%)$ & $2(1 \%)$ & \\
\hline Vaginal & $24(39 \%)$ & $125(56 \%)$ & $149(52 \%)$ & \\
\hline Cesarean & $37(61 \%)$ & $100(44 \%)$ & $137(48 \%)$ & \\
\hline Gestational DM & $3(5 \%)$ & $18(8 \%)$ & $21(7 \%)$ & 0.60 \\
\hline PROM & $6(10 \%)$ & $13(6 \%)$ & $19(6 \%)$ & 0.39 \\
\hline Preeclampsia & $9(15 \%)$ & $16(7 \%)$ & $25(9 \%)$ & 0.10 \\
\hline
\end{tabular}

Percentages are computed using the number of infants with a non-missing value.

IQR: interquartile range

${ }^{1}$ To compare the distribution of patient characteristics by antibiotic use, we employ chi-square tests. Similarly, we use Wilcoxon rank sum tests for continuous variables by antibiotic use.

${ }^{2}$ Percentages may not sum to $100 \%$.

Table 1C. Model Effects: Duration of Antibiotic Use

\begin{tabular}{lll}
\hline & Incidence Rate Ratio (95\% CI) & P-value \\
\hline Gestational age (per 1 week) & $0.98(0.93,1.03)$ & 0.48 \\
Admitted for sepsis & $1.46(1.09,1.95)$ & 0.010 \\
Admitted for RDS & $1.12(0.91,1.37)$ & 0.28 \\
Birth weight (3 vs. 2 kg) & $0.57(0.48,0.67)$ & $<0.001$ \\
Apgar at 1 minute (per 2 increase) & $0.80(0.70,0.91)$ & $<0.001$ \\
Vitamin D (per 2.6 increase) & $1.09(0.99,1.21)$ & 0.067 \\
PROM & $0.99(0.71,1.39)$ & 0.97 \\
Preeclampsia & $1.30(0.99,1.72)$ & 0.058 \\
Positive CRP & $1.48(1.02,2.15)$ & 0.040 \\
\hline
\end{tabular}

Table 1D. Model Effects: Odds of Death at Discharge (adjusted for gestational age)

\begin{tabular}{llll}
\hline & N & Odds Ratio $\mathbf{~ ( 9 5 \% ~ C I ) ~}$ & P-value \\
\hline Female & 317 & $1.80(0.63,5.14)$ & 0.27 \\
Admitted for sepsis & 317 & $1.10(0.24,4.97)$ & 0.90 \\
Admitted for RDS & 317 & $0.66(0.15,2.84)$ & 0.57 \\
Birth weight (3 vs. 2 kg) & 316 & $0.44(0.17,1.09)$ & 0.11 \\
Apgar at 1 minute (per 2 increase) & 178 & $0.26(0.12,0.57)$ & $<0.001$ \\
Apgar at 5 minutes (per 1 increase) & 201 & $0.56(0.37,0.83)$ & 0.004 \\
Days on antibiotics (per 5 days) & 312 & $0.73(0.48,1.13)$ & 0.16 \\
PROM & 308 & $0.69(0.10,4.71)$ & 0.71
\end{tabular}




\begin{tabular}{llll} 
Preeclampsia & 308 & $2.41(0.61,9.48)$ & 0.21 \\
Positive CRP & 288 & $10.52(2.25,49.16)$ & 0.003 \\
C-section (vs. vaginal) & 314 & $0.51(0.17,1.57)$ & 0.24 \\
Received mechanical ventilation & 316 & $776.00(57.50,10473.00)$ & $<0.001$ \\
Received oxyhood & 314 & $0.26(0.08,0.78)$ & 0.017 \\
Received nasal canula & 314 & $2.40(0.74,7.80)$ & 0.15 \\
\hline
\end{tabular}

${ }^{1}$ Adjusted for gestational age at birth.

\section{Copyrights}

Copyright for this article is retained by the author(s), with first publication rights granted to the journal.

This is an open-access article distributed under the terms and conditions of the Creative Commons Attribution license (http://creativecommons.org/licenses/by/3.0/). 